adenylate cyclase, presented new evidence that ST (the heat-stable low molecular weight enterotoxin(s) of $E$. coli, of which one example was characterised by D. Robertson (University of Kansas) as a peptide of 47 residues) acts by activating host cell (specifically intestinal epithelium) guanylate cyclase, while Gill indicated that the A-subunit of choleragen catalyses primarily the ADP-ribosylation of a GTP-binding protein which, according to observations by $Z$. Selinger, traps adenylate cyclase in its activated state. The subsequent events, catalysed by cyclicAMP or cyclicGMP, which lead to the increased electrolyte and fluid secretion, are still a grey area.

S. Falkow (University of Washington, Seattle) dynamically demonstrated the application of the newly-developing recombinant DNA technology to the solution of problems of pathogenesis, with the object of creating useful new microbial strains, rather than new pathogens, with evidence for the cloning of the ST and LT (heat-labile cholera-related enterotoxin) genes of $E$. coli. He supported the observations of F. $\emptyset_{\text {rskov (University of Copenha- }}$ gen) that the $E$. coli strains which are selected to become enteropathogens (that is, which can effectively receive tox plasmids and other essential virulence factors) may be restricted to a relatively small number of serotypes which happen to be relatively good genetic recipients. Some 'non-toxigenic' strains of $E$. coli of proven pathogenicity for man in volunteer studies may produce toxins which are active in other than the usual models, according to Levine. Other essential attributes of virulence, such as chemotaxis and adhesiveness leading to colonisation, were discussed by R. Freter (Michigan). J. Craig (New York) pointed out that cholera vibrios differ in the amount of toxin they elaborate in vitro under two different conditions of cultivation. The advantages and limitations of available techniques of recognising enterotoxins and their antibodies were discussed by Ö. Ouchterlony, who also presented a novel simple method of assay based upon changes in hydrophilic character of antigen-antibody complexes on plastic surfaces. The essential roles of the host cell glycolipid receptors, the $\mathrm{G}_{\mathrm{Ml}}$ ganglioside, elucidated by the pioneering observations of W. E. van Heyningen, were reviewed and extended by L. Svennerholm and J. Holmgren (Göteborg).

The possibilities of immunological intervention were high among the priorities of the Symposium. J. Feeley (Atlanta) extensively reviewed the scientifically controlled field studies of cholera vaccines composed of killed vibrios administered parenterally, and concluded, in accord with previous judgment, that the benefits derived did not justify the cost of vaccination. According to Barua, in consideration of these observations, requirements for cholera vaccination were removed from WHO regulations in 1973. Nevertheless there is the possibility of improvement, as brought out by more recent studies, presented by I. Joó (Hungary) and Pal, on alum-adjuvanted bacterial vaccines which seem to generate greater protection in the susceptible infants and children of endemic areas than previously tested un-adjuvanted vaccines. Although cholera toxoid administered parenterally offers no protection according to the field studies summarised by N. Ohtomo (Japan) and G. Curlin (Dacca), the synergistic protective effect observed experimentally with combinations of toxin antigen and somatic antigens in studies by A.-M. Svennerholm (Göteborg) is worth further consideration. She also indicated the potential value of assaying the antibody content of milk from immunised lactating women as a reflection of the local (intestinal) secretory antibody response. N. Pierce (Johns Hopkins University) reviewed extensively his observations on the kinetics of formation of antibody-containing cells in the lamina propria in response to a variety of combinations of orally and parenterally administered toxin antigens in experimental animal models and concluded that the nature of the antigen is important and that immunological suppression may result from some regimens. J. Holmgren (Göteborg) indicated that species differences may also be important, as illustrated by his studies in the orally immunised mouse model described earlier by Fujita and Finkelstein. J. Murphy (Harvard) reviewed genetic studies in his laboratory which have resulted so far in the isolation of two hypotoxinogenic mutants of strain 569B which produce reduced amounts of subunit $A$ and little, if any, subunit B.

That solid immunity against cholera is indeed feasible has been established conclusively by studies in volunteers as summarised by Levine. Volunteers convalescent from induced cholera were found to be virtually completely refractory to subsequent challenge with cholera vibrios of either the homologous or heterologous serotype. Therefore it is clear that the disease itself is an immunising process, although the mechanism(s) and the nature of the immunising antigen(s) - somatic antigens, enterotoxin, colonisation factors, flagella, outer membrane proteins, or a combination-are not yet known. Immunity to rechallenge after induced $E$. coli diarrhoea was markedly less effective.

In an earlier session, R. A. Finkelstein (University of Texas, Dallas) sum- marised the laboratory production and isolation of enterotoxins; elucidated criteria for defining enterotoxins and 'enterocytotoxins'; issued a caveat against the tendency to regard all newly described toxicities as toxins; and also reported the isolation with $T$. Honda, of a mutant of Vibrio cholerae which offers promise as a live vaccine against cholera and related diarrhoeas. Discussed further in the Immunology workshop, the mutant, called 'Texas Star', is avirulent in the sensitive infant rabbit model, has shown no signs of reversion, and seems to produce only the immunogenic $B$ (binding) portion of the cholera enterotoxin. It is hoped that the mutant will be shown to be innoccuous in volunteer studies and that it will simulate infection with virulent vibrios by producing solid immunity to cholera, and potentially to other diarrhoeas as well, by means of the common toxin $\mathbf{B}$ antigen. As a previous hypotoxinogenic mutant, M13, isolated in our laboratory, was harmless in volunteers and induced cholera immunity, this expectation may not be unrealistic.

In the immunology workshop it was emphasised that in volunter studies, which are an effective step toward vaccine development, the highest internationally acceptable ethical standards must be followed with regard to selection, education, and respect for the rights of volunteers.

Participants were reminded of how far we have come in a short time by the presence of one of the Indian investigators, S. N. De (Calcutta), who in the late $1950 \mathrm{~s}$ first showed that the symptoms of cholera could be produced in laboratory models by cell-free products of the cholera vibrio.

\section{Ultra-cold neutrons in superfluid helium}

from P. V. E. McClintock

THE first measurements of the rate at which ultra-cold neutrons are produced within a vessel of liquid helium, reported in a recent issue of Physics Letters (66A, 469; 1978), strongly support the earlier suggestion by Golub and Pendlebury (Physics Letters 62A, 337 ; 1977) that it might be possible to fill a neutron bottle with trapped neutrons to an unprecedentedly high density if superfluid ${ }^{4} \mathrm{He}$ were used as the internal medium. The production measurements were carried out in Grenoble at the Institut LaueLangevin by P. Ageron and W. Mampe (ILL) in collaboration with $\mathrm{R}$. Golub and J. M. Pendlebury (Sussex University).

Ultra-cold neutrons (UCN) are neu- 
trons of such low energy that they are totally reflected from material surfaces at all angles of incidence, so that it becomes possible to confine them inside a suitably designed container (neutron bottle), where experiments can then be conducted in a relatively leisurely fashion-compared to neutron beam experiments where the time of observation is restricted to about $1 / 30 \mathrm{~s}$ at most-to elucidate their properties. If they could be trapped at sufficient density for periods comparable with their lifetime (neutrons decay by $\beta$-emission in about 15 minutes), then it might become possible to determine certain fundamental constants, such as the electric dipole moment of the neutron for which there are various theoretical predictions but, as yet, no positive measurements. The main difficulty is that the present technology available for UCN production, exploiting the very low energy Maxwellian tail of the thermal distribution of neutrons within a nuclear reactor, leads to trapped UCN densities of only about $1 \mathrm{~cm}^{-3}$, which is not really adequate for the proposed experiments.

Golub and Pendlebury's suggestion amounted to a completely different technique by which UCN might be produced: by down-scattering in liquid helium of neutrons with an initial velocity of about $450 \mathrm{~m} \mathrm{~s}^{-1}$. They pointed out that, when a neutron of this particular velocity travels through a bath of superfluid ${ }^{4} \mathrm{He}$, there is a finite probability that it may spontaneously create a thermal excitation (in effect, a single quantum of sound) and, in doing so, lose almost all its initial energy. In its final state it will be an ultra-cold neutron, moving at less than $5 \mathrm{~ms}^{-1}$, and unable to escape from the container. Their proposed UCN source consists, therefore, of a container of superfluid ${ }^{4} \mathrm{He}$ whose walls are transparent to neutrons with velocity of $450 \mathrm{~ms}^{-1}$, placed in a beam containing a relatively large proportion of neutrons of this velocity. Most of the neutrons will pass straight through and out the other side, but a certain number will create excitations, enter the UCN region and become trapped.

The UCN density attainable by this method clearly depends on a balance between the production rate and the loss rate. Losses of UCN from the bottle will occur inevitably through $\beta$-decay but, in addition, can also arise as the result of up-scattering by thermal excitations (the converse of the production mechanism), absorption by impurities in the ${ }^{4} \mathrm{He}$, or inelastic scattering from the walls. The latter phenomenon has restricted the storage times achieved in evacuated roomtemperature bottles to about $30 \mathrm{~s}$, but it is expected to become much less important at the temperatures over
$1 \mathrm{~K}$ where the helium filled bottle will be operated. The most potent neutron absorber likely to be present in the liquid is the other isotope of helium, ${ }^{3} \mathrm{He}$ : it will be necessary to reduce the ${ }^{3} \mathrm{He}$ isotopic impurity content to a level about four or five orders of magnitude below that (a few parts in $10^{7}$ ) found in ordinary commercial ${ }^{4} \mathrm{He}$. Fortunately, however, purities even greater than this can quite readily be achieved using the heatflush isotopic separation method recently developed at Lancaster, and neutron absorbtion within the body of the liquid is not therefore expected to be a problem. Neither should up-scattering processes since, provided the hottle is operated at a temperature below $1 \mathrm{~K}$, the number of excitations with the appropriate energy to initiate such events will be quite negligible. The main unknown factor has been the production rate and, although Golub and Pendlebury did their best to estimate it, the calculation was by no means sraightforward and it incorporated a number of assumptions which could have been in error. Hence the importance of the work now being reported from the ILL, which constitutes the first experimental measurement of the UCN production rate in superfluid helium.

The arrangement used was extremely simple. A vessel of liquid ${ }^{4} \mathrm{He}$, whose temperature could be reduced by pumping, was placed in a horizontal neutron beam. Ordinary commercial ${ }^{4} \mathrm{He}$ was used, and so no provision was made for storing any UCN which might be produced. Instead, a neutron guide tube was provided to lead the UCN out of the liquid helium to an external neutron detector. The guide incorporated five right-angled bends to enable neutrons of higher energy to escape through its walls and thus to ensure that only the UCN could reach the detector and be counted.

In the event, the neutron counting rate increased rapidly to about $5 \mathrm{~s}^{-1}$ when the temperature of the liquid was reduced below its superfluid transition temperature at $2.2 \mathrm{~K}$. When a $0.5 \mathrm{~mm}$ thick stainless steel plate, totally opaque to UCN but partially transparent to neutrons of higher energy, was placed so as to block the guide tube, the counting rate fell to the background level of $0.5 \mathrm{~s}^{-1}$; but it returned to its former value again as soon as the plate was removed. Some 24 hours later, as the last of the liquid helium boiled away, the rate fell once more to the background level. It seems virtually certain, therefore, that the detected neutrons were UCN produced in the liquid through the mechanism proposed by Golub and Pendlebury. Furthermore, the measured production rate was found to be in remarkably good agreement with the value which they had calculated.

This is a very encouraging result. It implies that the fullscale neutron confinement cryostat now being planned at the ILL, which will incorporate a sample of isotopically ultra-pure liquid ${ }^{4} \mathrm{He}$, is likely to store UCN at densities at least sixty times larger than have been attained by conventional methods -and perhaps a thousand times larger, if the problem of wall losses can be overcome.

P. V. E. McClintock is in the Physics Department at the University of Lancaster.

\section{Progress in Fusion Research}

from a Correspondent

ToKAMAK rules. That was the essential message from the 7 th International Conference on Plasma Physics and Controlled Nuclear Fusion Research held at Innsbruck from 23-30 August last. The results from the large (PLT) tokamak at Princeton (USA) were presented and discussed in some detail. In particular the scientific basis for the widely publicised claim that a temperature of 60 million degrees had been reached was carefully examined and finally accepted. The discussions highlighted the difficulties of unambiguous measurement of ion temperatures in such plasmas. Indeed it can be argued that the obsession with 'temperature' is a hangover from the Zeta days and that in these plasmas, particularly those heated by powerful neutral beams, the single crucial number is the ratio $Q$ of the energy released in fusion reactions to the energy input. In the Princeton experiment with $1.7 \mathrm{MW}$ of neutral deuterium beam power into a deuterium plasma, a neutron emission of $4 \times 10^{13}$ neutrons $\mathrm{s}^{-1}$ was measured; at $\sim 3 \mathrm{MeV}$ per reaction, one can calculate that this corresponds to $\sim 20 \mathrm{~W}$ of fusion power and hence to $Q \sim 10^{-5}$. If a tritium beam had been used, then the more favourable cross-section and larger energy release would have increased $Q$ to $\sim 5 \times 10^{-3}$. The new tokamak TFTR at present under construction at Princeton is designed to achieve $Q>1$ through the reduction in diffusive losses by the use of larger dimensions. In the light of the new results its chances of success look good. For a power reactor $Q \sim 20$ would be needed.

Results from other tokamak experiments around the world showed an increasing ability to reduce the contamination of the plasma by impurities. With cleaner plasmas it is possible to reach higher densities than before and to increase the ratio of toroidal plasma current to toroidal magnetic field. Thus 